\title{
Mechanisms of transmembrane cation transport studied by nuclear magnetic resonance spectroscopy
}

\author{
M. B. SANKARAM, B. P. SHASTRI and K. R. K. EASWARAN \\ Molecular Biophysics Unit, Indian Institute of Science, Bangalore 560 012, India

\begin{abstract}
Several molecules like ionophores, vitamins, ion-binding cyclic peptides, acidic phospholipids, surfactants are known to expose the inner side of vesicles, to the externally added cations. Whereas ionophores and certain other systems bring about these changes by a selective transport (influx) of the cation by specialized mechanisms known as the carrier and channel mechanism, other systems cause lysis and vesicle fusion. These systems have been successfully studied using ${ }^{1} \mathrm{H},{ }^{31} \mathrm{P}$ and ${ }^{13} \mathrm{C}$ nuclear magnetic resonance spectroscopy after the demonstration, fifteen years ago, of the ability of paramagnetic lanthanide ions to distinguish the inside of the vesicle from the outside. The results of these 'nuclear magnetic resonance kinetics' experiments are reviewed.
\end{abstract}

Keywords. Nuclear magnetic resonance spectroscopy; membrane transport; ionophores; vesicles; transport kinetics.

\section{Introduction}

The cell plasma membrane is a vectorial barrier which modulates the interaction of the cytoplasmic substances with the external medium. In addition to being primarily responsible for the order in living systems, the membrane gets the cell going by maintaining appropriate gradients across it. Inquiries into the nature of the mechanisms underlying the permeabilities of solutes have run parallel to those probing into the structure of the membrane itself. The essential issue was posed soon after the recognition of the lipoidal nature of the membrane and the proposal of a bilayer model for the membrane (Davson, 1962).The issue to be settled was-what makes the small, charged ions such as $\mathrm{K}^{+}$and $\mathrm{Ca}^{2+}$ go through an essentially apolar medium formed by the hydrocarbon chains of the lipids. It was soon recognized that specialized mechanisms called membrane channels might be operative (Hober, 1945). This idea eventually was set on a firm ground following the discovery of molecules that transport cations across bilayer lipid membranes (BLMs), called ionophores (Pressman, 1976).

\section{Ionophores as carriers and channels}

The bilayer lipid membranes are very good insulators. Relatively, cell membranes are poor insulators. A logical conclusion that follows from this observation is that

\footnotetext{
Abbreviations used: BLM, Bilayer lipid membrane; NMR, nuclear magnetic resonance; EDTA, ethylene diamine tetraacetic acid; DPA, dipicolinate; NTA, nitrilotriacetate; PPPi, tripolyphosphate.
} 
membrane-associated proteins act as 'mediators' for cation translocation across membranes (Cole, 1968). Whereas difficulties in isolating the membrane-proteins hampered any further development, studies on chemically (by ionophores) modified lipid bilayers helped in understanding the possible mechanisms underlying the phenomenon of transmembrane cation transport.

Ionophores are a class of compounds isolated from microorganisms. Peptides, depsipeptides, polyethers and carboxylic acids in both cyclic and acyclic form are the commonest known ionophores, the most important of them being valinomycin, lasalocid, A23187 and gramicidin A (Ovchinnikov et al., 1974). These molecules of microbial origin enchance the cation permeabilities of bilayer lipid membranes. Chemical structures of some of these ionophores are shown in figure 1.The ionophoretic behaviour of these molecules, as studied by electrical measurements on BLMs, was classified into two categories, namely channels and carriers.

According to a widely accepted model, known as the carrier mechanism, the cation finds its way from the aqueous medium on one side of the membrane-water interfacial region, through the two-dimensional fluid called the bilayer membrane, down a flexibility gradient of the acyl chains to the interior of the membrane. An almost exact reversal of the steps takes it to the other side of the membrane. This mechanism is best, exemplified by valinomycin, lasalocid, nonactin and A23187. A variant of this
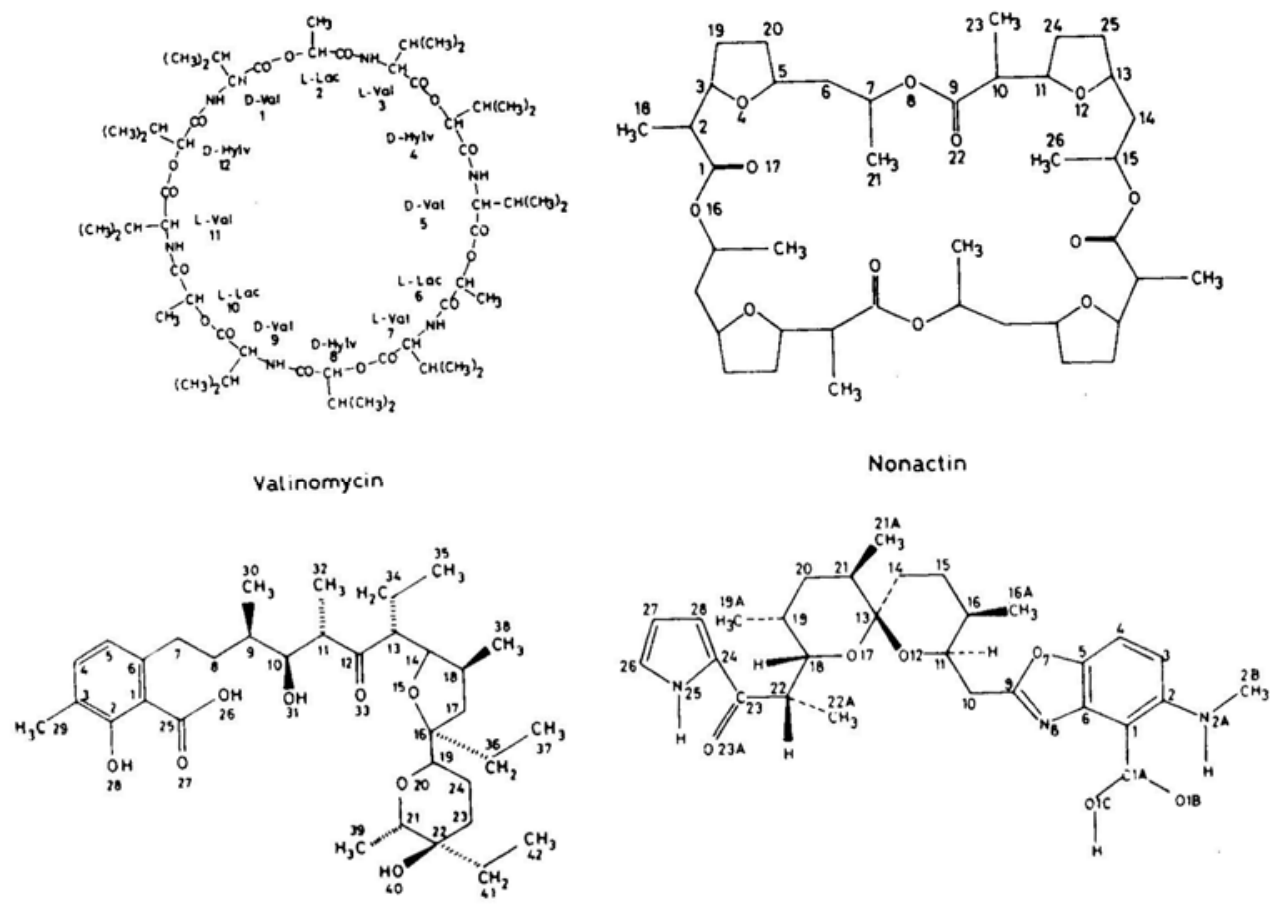

Lasalocid

Figure 1. Chemical structures of ionophores. 
mechanism is also known, called the relay carrier mechanism, (Ivanov, 1975) where the cation is handed over from one ionophore to another in the membrane. Obviously, the efficiency of this mechanism is dependent on the probability that two ionophores molecules meet in the membrane and hence would be observable only at high local ionophore concentrations.

Channels are more efficiency than carriers. A channel forming molecule spans the membrane between the interfaces, with a hydrophobic exterior and a hydrophilic interior which the cations can pass through. Whereas per journey of the carrier from one side to the other and back one cation is transported, one opening of the channel results in an entry of a large number of cations. Gramicidin A and alamethicin are widely studied by the BLM electrical measurements (Urry et al., 1980; Boheim et al., 1980). Cations are not bound by channels as strongly as in the case of carriers. The carrier and channel mechanisms are illustrated in figure 2.

Studies aimed at understanding the ionophoretic abilities of carriers revealed a propensity of them to form very stable carrier-cation complexes in organic solvents. Extensive investigations of this propensity of carriers like valinomycin, nonactin, lasalocid and A23187 to complex with mono-, di- and tri-valent cations led to the identification of equimolar and ion-sandwich complexes with possible functional significance (Ovchinnikov et al., 1974; Easwaran, 1985; Vishwanath and Easwaran, 1981,1982,1985; Shastri and Easwaran, 1984).The conformations of such complexes formed by valinomycin are shown in figure 3. Irrespective of the stoichiometry of the complex, nature of the ionophore and the cation, several generalizations can be drawn from these observations. All these complexes have non-polar exteriors and effectively shield the cation from the surrounding environment. This is a primary requirement for a molecule to act as a cation mediator by lowering the activation energy barrier. In medium to polar solvents, the cation is bound by a part of the molecule with the rest of the molecule having a non-polar exterior. One would expect this to be the case when the cation is being complexed by the membrane-bound carrier.

Conductances of most carrier modified BLMs showed a linear dependence on both cation and carrier concentration suggesting that the equimolar complexes are the most

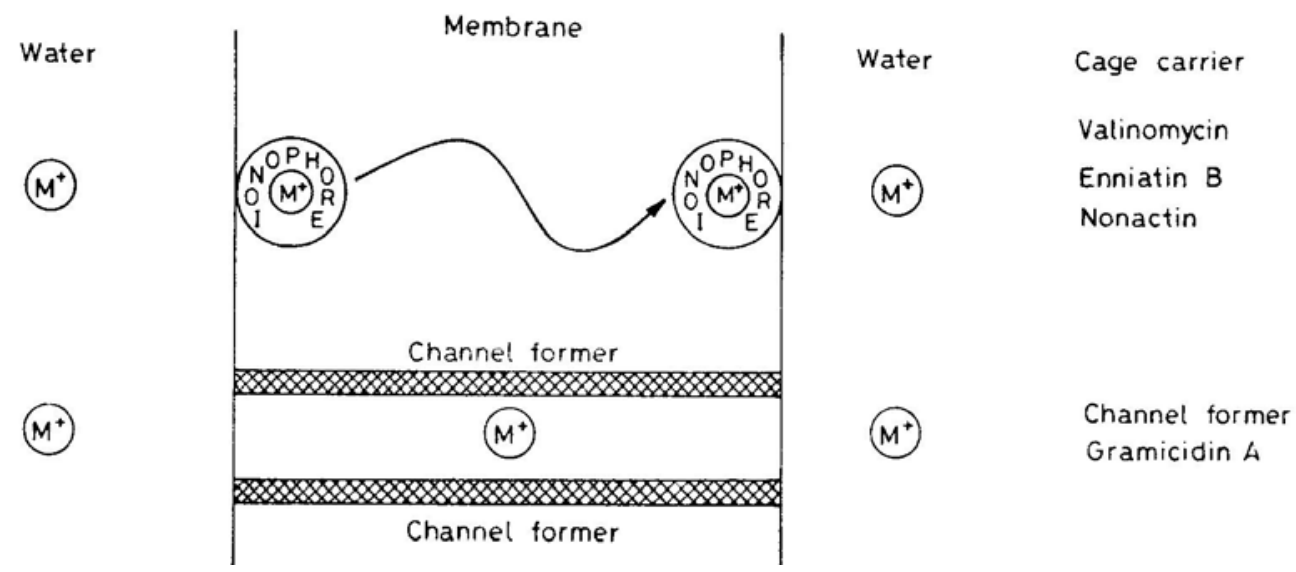

Figure 2. Mechanisms of cation transport. 


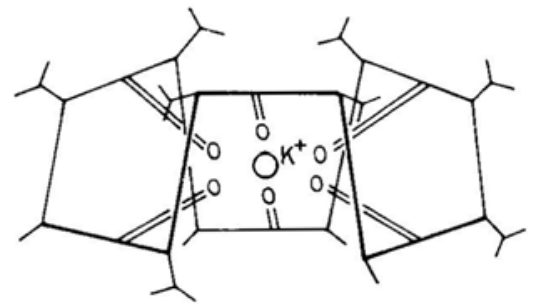

(a)

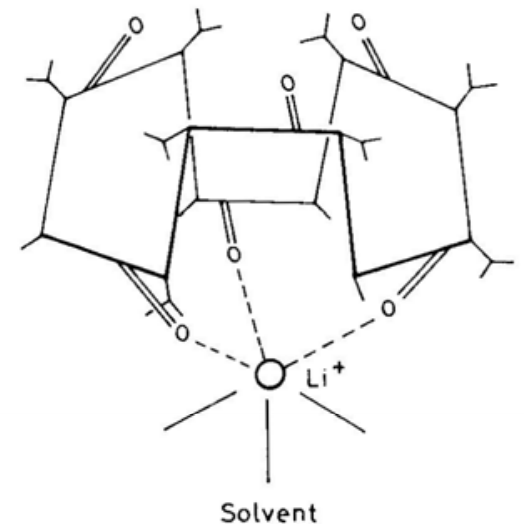

(b)

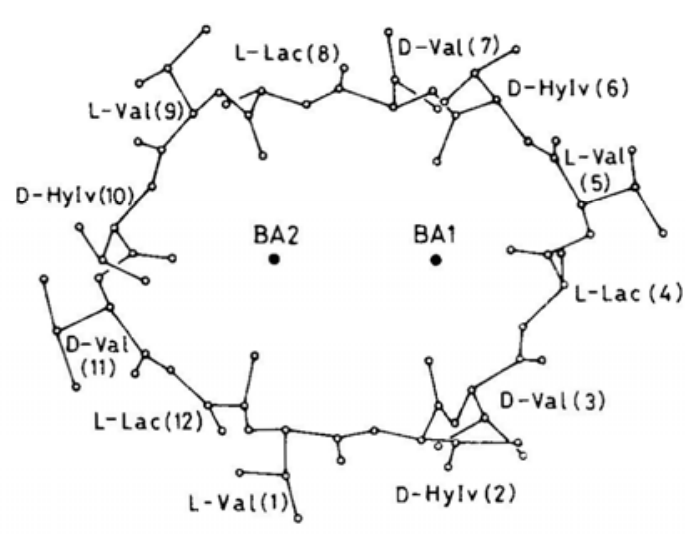

(d)

(c)

Figure 3. Conformations of valinomycin-cation complexes (a), 1:1 $\mathrm{K}^{+}$type complex; (b), 1:1 peripheral complex; (c), 2:1 ion-sandwich complex and (d), 1:2 open complex.

likely transporting species (Grell et al·, 1974) - Non-linear dependences indicating cation mediation by both ion-sandwich and equimolar complexes are also known although a one-to-one correspondence between the stoichiometry of the cation-carrier complex in solution and the stoichiometry of the transporting species is not always found (Szabo et $a l \cdot, 1975) \cdot$ These apparent inconsistencies have been traced to a competition between several relevant rate processes (Hladky, 1979) and coparticipation of both ionsandwich and equimolar complexes (Shekar et al·, 1984).

\section{Use of vesicles as membrane models}

A detailed description of the mediated transport in terms of the kinetic rate constants of the complexation, diffusion and decomplexation reactions has been given mainly by 
electrical measurements on BLMs (Hladky, 1979). The BLM is a flat bilayer which has been very convenient to use mainly because it allow the experimenter to control the aqueous media on either side of it. However, they lack the curvature the spherical cells have and frequently contain organic solvents due to the inherent nature of their preparation. Vesicles are, in this respect, better model systems. The size of the vesicles can be varied at will and also their chemical composition accurately determined. Electrical measurements are, however, extremely difficulty to perform on these systems.

\section{Effect of lanthanides on the NMR spectra of vesicles}

In recent years, NMR techniques have been developed to monitor the mediated transport across vesicles which have been used very successfully. In 1970, Bystrov and coworkers (Bergelson et al., 1970; Bystrov et al., 1971) have shown that paramagnetic lanthanide ions can be used to distinguish between the two sides of the vesicles. The method has been applied to a large number of cases since then.

In spite of the oligomeric structure of vesicles, NMR spectra of vesicles contain signals from the most important regions of the lipid structure. The ${ }^{1} \mathrm{H}$ NMR spectra show effective, time-averaged signals from the head group cholines, hydrocarbon methylene chains and the terminal methyl groups. At very high magnetic fields, the asymmetry between the two halves is reflected in the NMR spectra. Addition of $\mathrm{Ln}^{3+}$ makes this asymmetry more pronounced (Bergelson, 1978) by complexing with the phosphates from the headgroups jetting out of the side where it is added. This binding results in large chemical shift changes in the choline protons $\left({ }^{1} \mathrm{H}\right.$ NMR) phosphate $\left({ }^{31} \mathrm{P}\right.$ NMR) and choline methyl carbon $\left({ }^{13} \mathrm{C}\right.$ NMR) signals. Depending on the nature of the lanthanide ion, the choline signals will either be shifted or broadened. The effect of lanthanide addition on the $1 \mathrm{H}$ NMR spectra of vesicles is summarized in table 1.

Table 1. Effect of $\mathrm{Ln}^{3+}$ ions on the NMR parameters of vesicles*.

\begin{tabular}{lccc}
\hline $\mathrm{Ln}^{3+}$ & $\begin{array}{c}\text { Shift } \\
(\Delta \delta)\end{array}$ & $\begin{array}{c}\text { Line width } \\
(\mathrm{Hz})\end{array}$ & $\begin{array}{c}\text { Concentration } \\
\text { of } \mathrm{Ln}^{3+}(\mathrm{mM})\end{array}$ \\
\hline- & 0 & $4 \cdot 2$ & - \\
$\mathrm{Pr}^{3+}$ & $0 \cdot 426$ & $9 \cdot 4$ & 10 \\
$\mathrm{Nd}^{3+}$ & $0 \cdot 261$ & 6.8 & 10 \\
$\mathrm{Eu}^{3+}$ & -0.187 & 6.5 & 10 \\
$\mathrm{~Tb}^{3+}$ & 0.810 & 23.5 & 1 \\
$\mathrm{Dy}^{3+}$ & $1 \cdot 1$ & 32.0 & 1 \\
$\mathrm{Ho}^{3+}$ & $0 \cdot 457$ & 13.0 & 1 \\
$\mathrm{Tm}^{3+}$ & -0.52 & 17.0 & 10 \\
$\mathrm{Yb}^{3+}$ & -0.305 & 10.4 & 1 \\
\hline
\end{tabular}

* Taken from Bergelson, 1978. 


\section{Kinetics of cation transport by NMR}

Vesicles are impermeable to lanthanide ions. When an ionophore is added, time dependent changes in the line widths, chemical shifts and intensities of the choline signals are observed typical of the mechanism of transport. As mediated transport begins, the lanthanide ions enter the vesicles resulting in a shift of the inner (if the cation were added outside) head group resonance and a change in its shape. The time course of these changes contain much information on the actual transport mechanism. Processes by which ions enter the vesicles one or a few at a time are easily distinguishable from those by which they enter in large bursts. Also, the line shapes depend on the competition of the intervesicular mediator exchange with transport. A detailed analysis of the NMR kinetics experiments has been made on these lines (Ting et al., 1981).

\section{Cation transport by carriers}

Several carriers, mainly the carboxylic group ionophores, have been studied by ${ }^{1} \mathrm{H}$ and ${ }^{31} \mathrm{P}$ NMR kinetics. Results on the carrier type ionophores are summarized in table 2 . It is shown using ${ }^{1} \mathrm{H}$ NMR that lasalocid transports $\operatorname{Pr}^{3+}$ via a 2:1 complex (Fernandez et al., 1973) In a similar study, $\mathrm{Mn}^{2}{ }^{+}$mediation by both $2: 1$ and $1: 1$ complexes has been observed (Degani et al., 1981). At low ratios of lasalocid to vesicle, transport via a 1:1 complex predominates. As the concentration of lasalocid is increased, a 2:1 complex acts as the transporting species. As discussed above, this evidence could be taken to support a relay carrier mechanism.

A23187 has also been shown to enhance $\operatorname{Pr}^{3+}$ permeabilities. The evidence is in favour of a 1:1 complex acting as the transporting species (Hunt et al., 1978). Our detailed kinetic analysis of the mediation of lanthanides by A23187 shows that as one goes along the lanthanide series from $\mathrm{Pr}^{3+}$ to $\mathrm{Lu}^{3+}$, the rates of transport decrease (Sankaram and Easwaran, 1984). These results are very well correlated with the cation complexing abilities of carboxylic ionophores. The time dependence of the choline region ${ }^{1} \mathrm{H}$ NMR spectra of lasalocid mediated $\mathrm{Eu}^{3+}$ transport across DPPC vesicles is shown in figure 4.

In addition to these two most widely studied ionophores, NMR kinetics of $\operatorname{Pr}^{3+}$

Table 2. Summary of NMR kinetics of carriers.

\begin{tabular}{|c|c|c|c|c|}
\hline Carrier & Cation & Lipid & $\begin{array}{c}\text { Transporting } \\
\text { species }\end{array}$ & Reference \\
\hline \multirow[t]{2}{*}{ Lasalocid A } & $\mathbf{P r}^{3+}$ & EYL & $2: 1$ & Fernandez et al., 1973 \\
\hline & $\mathrm{Mn}^{2+}$ & DPPC & $1: 1,2: 1$ & Degani et al., 1981 \\
\hline A23187 & $\left.\begin{array}{l}\mathrm{Pr}^{3+}, \mathrm{Eu}^{3+} \\
\text { and } \mathrm{Nd}^{3+}\end{array}\right\}$ & $\begin{array}{l}\text { DPPC } \\
\text { DMPC. }\end{array}$ & $2: 1$ & Shastri, 1985 \\
\hline Etheromycin & $\mathrm{Pr}^{3+}$ & EYL & $2: 1$ & Donis et al., 1981 \\
\hline M139603 & $\mathrm{Pr}^{3+}$ & EYL & $1: 1$ & Grandjean and Laszlo, 1984 \\
\hline$X-14547 \mathrm{~A}$ & $\mathbf{P r}^{3+}$ & EYL & $1: 1$ & Grandjean and Laszlo, 1984 \\
\hline Tetronomycin & $\operatorname{Pr}^{3+}$ & EYL & $1: 1$ & Grandjean and Laszlo, 1984 \\
\hline Narasin & $\mathrm{Pr}^{3+}$ & EYL & $1: 1$ & Grandjean and Laszlo, 1984 \\
\hline
\end{tabular}




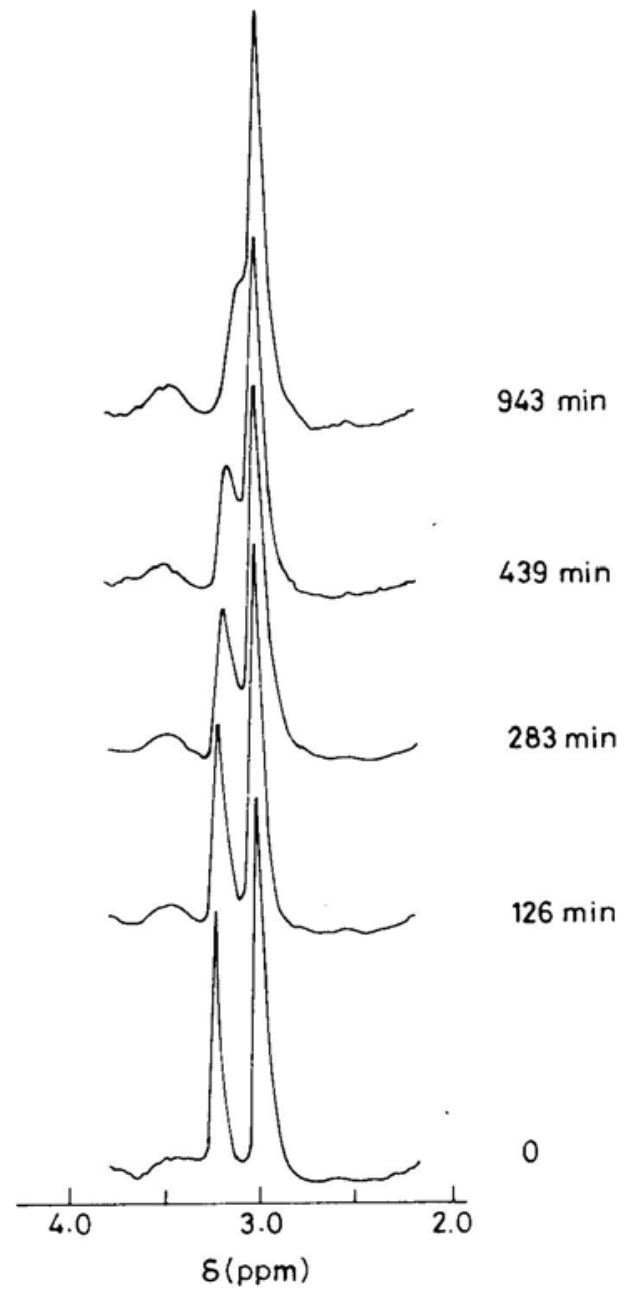

Figure 4. Time dependence of the choline region ${ }^{1} \mathrm{H}$ NMR spectra of lasalocid A mediated $\mathrm{Ei}^{3+}$ transport across phospholipid vesicles.

transport by etheromycin (Donis et al., 1981), M139603, X-14547A, tetronomycin and narasin (Grandjean and Laszlo, 1984) have been studied. These carboxylic ionophores exchange protons with the lanthanide, exhibiting an antiport mechanism. The activation energies measured for $\mathrm{Pr}^{3+}$ transport are $120 \mathrm{~kJ} \mathrm{~mol}^{-1}$ (Donis et al., 1981) for etheromycin, $105 \mathrm{~kJ} \mathrm{~mol}^{-1}$ for lasalocid (Grandjean and Laszlo 1982), and $116 \mathrm{~kJ} \mathrm{~mol}^{-1}$ for A23187 (Hunt et al., 1978). These values are fully consistent with carrier-mediated transport, for which values in the range of $90-130 \mathrm{~kJ} \mathrm{~mol}^{-1}$ are obtained.

Whereas carboxylic ionophores act as very good carriers for lanthanide transport across vesicles, neutral ionophores show kinetics. Thus, it has been observed that several cyclic and acyclic polyethers lacking an ionizable carboxylic acid group show 
rates several orders of magnitude slower than those for carboxylic ionophores (Donis et al., 1981). Observable rates are obtained only at very high ionophore to vesicle ratios and the observed dependence of the rate constants on the ionophore concentration is not monotonic. Use of these ligands at high concentrations leads to perturbations in the membrane structure, providing extraneous mechanisms for ion penetration. The lack of effective carrier activity in the case of these neutral ionophores is due to their inability to antiport protons. When $\operatorname{Pr}^{3+}$ ions are complexed by the ionophore on one side, the counter ions get stuck to the choline head groups. To achieve electroneutrality, protons could be antiported which is effectively done if the ionophore contains an ionizable carboxylic acid group. Evidence for this kind of antiport mechanism comes from the observation of enhanced rates of $\operatorname{Pr}^{3+}$ transport by neutral ionophores in the presence of hydrophobic anions like picrate.

\section{Synergism}

A remarkable positive cooperativity has been observed in the transport of $\operatorname{Pr}^{3+}$ ions across phosphatidylcholine vesicles, when two carboxylic ionophores are present in the lipid bilayer. It has been found that when etheromycin, monensin (Grandjean and Laszlo, 1982) narasin, tetronomycin, A23187, X206, X-14547A and M139603 (Grandjean and Laszlo, 1984) are used along with lasalocid, they exhibit a pronounced synergism, amounting to rate enhancements by a factor of 3 to 12. Similar synergism has been found with the pairs etheromycin and narasin, etheromycin and X-14547A and monensin and X-14547A (Grandjean and Laszlo, 1984). The rate enhancements have been shown to be due to a facilitated antiport by the hybrid complexes and not due to a greater thermodynamic stability of the hybrid complex as compared to the individual 2:1 complexes. Evidence for this argument comes from (a) the observation of enhanced rates when picrate is the counter ion supporting the proton-cation exchange to maintain electroneutrality and (b) absence of any substantial and additional cation-ionophore interactions as revealed by ${ }^{13} \mathrm{C} N \mathrm{NR}$.

\section{Transport by amphipaths}

Certain amphipaths like triton $\mathrm{X}-100$, bile salts and lysolecithin also induce cation transport across vesicles. When $\operatorname{Pr}^{3+}$ is carried across phosphatidylcholine vesicles by bile salts (Hunt, 1980), the rise in intravesicular concentrations causes the signal from inside head groups to move downfield toward the signal from the outside headgroup. The stoichiometry has been calculated to be $4: 1$ which led to a suggestion of inverted micellar intermediates (formed from four molecules of the bile salt) as the transporting species. In the presence of $40 \%$ cholesterol, addition of deoxycholate and chenodeoxycholate results in a NMR spectral behaviour different from that observed for carries (Hunt and Jawaharlal, 1980). A new peak upfield to the outer choline signal progressively appears at the expense of the inner choline signal. The new peak is broad suggesting its origin as the $\operatorname{Pr}^{3+}$ enters the vesicles and increases in intensity and the concomitant decrease in the free inner choline head group signal intensity suggests a time-dependent change from a sample in which all vesicles have no $\operatorname{Pr}^{3+}$, with the 
mechanism allowing a gated pulse of $\operatorname{Pr}^{3+}$ across the bilayer. A theoretical analysis of this behaviour has been made and the mechanism has been identified to be a slow mediator exchange (Ting et al., 1981).

With lysolecithin, below the lipid phase transition temperature, a very fast $\operatorname{Pr}^{3+}$ transport has been observed (Lee and Chan, 1977). At these temperatures, the host lipid phase separates leading to formation of patches of lysolecithin molecules within the lecithin matrix. These clusters are long lived and sufficiently perturb the membrane structure to serve as channels for rapid ion translocation. Above the phase transition temperature, four lysolecithin molecules have been proposed to form a channel. Activation energies of $54 \mathrm{~kJ} \mathrm{~mol}^{-1}$ for palmitoyl lysolecithin and $22.6 \mathrm{~kJ} \mathrm{~mol}^{-1}$ for myristoyl lysolecithin have been considered to be evidences to rule out any carrier mechanism.

\section{Cation transport by channels}

An ideal channel would exhibit an 'all or nothing' process in which either a sufficient number of cations to saturate the inner pools enter the vesicle when the channel is open or nothing enters at all when the channel is closed (Ting et al., 1981). This results in a growth of vesicles with cation-saturated pools at the expense of cation-free pools. The corresponding spectra would show a growth of a signal from the head groups of the inner side of the vesicle that have received all the cations they can with a concomitant decrease on the intensity of the signal from inner head groups of vesicles containing no cations (figure 5). Such spectral changes have been observed for light induced $\mathrm{Eu}^{3+}$ transport by rhodopsin (O'Brien et al., 1977), thermally induced $\mathrm{Eu}^{3+}$ and $\mathrm{Nd}^{3+}$ permeation (Lawaczeck et al., 1977), sonication induced $\mathrm{Pr}^{3+}$ and $\mathrm{Eu}^{3+}$ permeation (Ting et al., 1981) and cardiolipin induced $\mathrm{Pr}^{3+}$ translocation (Sankaram et al., 1984). Formation of transmembrane channels providing a hydrophilic interior which cations can pass through has been observed only in the case of rhodopsin and cardiolipin. In the case of thermally induced changes, the rate of relative number of inward facing lipid molecules which became exposed to extravesicularly added lanthanide ions has been found to be a function of the inverse lipid concentration. This rules out any diffusion, pore activation or rupture of vesicle walls and suggests a dynamic equilibrium between the various states of the lipid molecules in water such as monomers, micelles, vesicles and multilamellar vesicles as an explanation for the observed changes. However, in the case of sonication induced changes, each pulse of sonic power applied is equivalent to an opening of a channel which pumps cations in bursts.

There are certain border line cases where the channel is not stable for a length of time necessary for the required number of cations to go through. This would result in carrier like kinetics but the kinetic analysis would suggest molecularities for the transporting species which can be modelled to be channels (Ting et al., 1981). Most of the surfactant and lysolecithin mediated transport experiments fall into this category. We have recently shown that an ion-binding cyclic hexapeptide cyclo (L-pro-gly) 3 forms hexameric channels spanning the membrane for transporting $\operatorname{Pr}^{3+}$ but exhibits carrier like kinetics (Sankaram, 1983). 


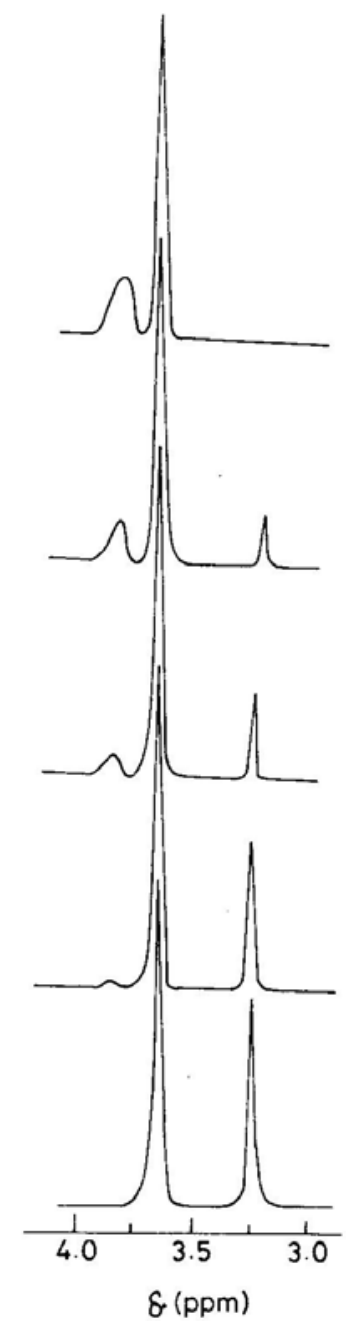

Figure 5. Time dependence of the choline region ${ }_{1} \mathrm{H}$ NMR spectra for an 'all or nothing' process.

\section{Vitamins, antibiotics and other systems}

In many cases, NMR kinetics experiments have been done on lanthanide translocation without detailed analyses to propose molecular models for the transporting species. Vitamin E, phytanic acid, phytol (Cushley and Forrest, 1977) have been shown to induce exposure of inner cholines to externally added $\operatorname{Pr}^{3+}$. The polyene macrolide antibiotic, nystatin (Pierce et al., 1978) has been shown to exhibit similar behaviour with three molecules of the antibiotic required to transport one $\operatorname{Pr}^{3+}$. The changes have been ascribed to a severe disruption of the membrane structure by nystatin. Reduction in the rate of transport has been observed in the presence of cholesterol which supported this argument. Glycophorin (Gerristen et al., 1979) has been shown to expose the inner head 
groups to externally added $\mathrm{Dy}^{3+}$ using ${ }^{13} \mathrm{C}$ NMR. Alamethicin (Lau and Chan, 1975) has been shown to induce membrane fusion when added to vesicles containing $\mathrm{Eu}^{3+}$. Alamethicin has been shown to traverse the membrane taking $\mathrm{Eu}^{3+}$ as a counter ion along with it. When alamethicin and $\mathrm{Eu}^{3+}$ are present in the intravesicular space, $\mathrm{Eu}^{3+}$ efflux has not been observed.

\section{Transport kinetics by cation NMR}

Several hydrophilic ligands like Dy(EDTA) ${ }^{-}, \operatorname{Dy}(\mathrm{DPA})_{3^{-}}^{3^{-}}, \mathrm{Dy}(\mathrm{NTA})_{2}^{3-}$ (Pike and Springer, 1982), Gd(EDTA) ${ }^{-}$(Degani and Elgavish, 1978), Dy(PPPi) ${ }_{2}^{3-}$ (Gupta and Gupta, 1982) and Dy $\left[\mathrm{N}\left(\mathrm{CH}_{2} \mathrm{CH}_{2}\right)_{3}\right]_{2}^{3-}$ (Pike et al., 1982) have been synthesized which complex with $\mathrm{Na}^{+}$and $\mathrm{Li}^{+}$and broaden their signals. This method has permitted the use of ${ }^{23} \mathrm{Na}$ and ${ }^{7} \mathrm{Li} \mathrm{NMR}$ to monitor their transport kinetics. In vivo experiments on human erythrocytes and the northern frog rana pipiens have been shown to be feasible employing these reagents (Pike et al., 1982; Pike and Springer, 1982). It has been demonstrated by ${ }^{23} \mathrm{Na} \mathrm{NMR}$ that gramicidin A exhibits an 'all or nothing' behaviour.

\section{References}

Bergelson, L. D. (1978) Meth. Membrane Biol., 9, 275.

Bergelson, L. D., Barsukov, L. I., Dubrovina, N. I. and Bystrov, V. F. (1970) Dikl. Acad. Nauk (USSR), 194, 708.

Boheim, G., Hanke, W. and Eibl, H. (1980) Proc. Natl. Acad. Sci. USA, 77, 3403.

Bystrov, V. F., Shapiro, Y. E., Biktorov, A. V., Barsukov, L. I. and Bergelson, L. D. (1971)FEBS Lett., 25,337.

Cole, K. S. (1968) Membranes, ions and impulses (Berkeley, University of California Press).

Cushley, R. J. and Forrest, B. J. (1977) Can. J. Chem., 55, 220.

Davson, H. (1962) Circulation, 26, 1022.

Degani, H. and Elgavish, G. A. (1978) FEBS Lett., 90, 357.

Degani, H., Simon, S. and McLaughlin, A. C. (1981) Biochim. Biophys. Acta, 646, 320.

Donis, J., Grandjean, J., Gresjean, A. and Laszlo, P. (1981) Biochem. Biophys. Res. Commun., 102, 690.

Easwaran, K. R K. (1985) in Metal ions in biological systems, (ed. H. Sigel) (New York: Marcel-Dekker) Vol. 19, Chapter 5 (in press).

Fernandez, M. S., Celis, H. and Montal, M. (1973) Biochim. Biophys. Acta, 323, 600; van Deened, L. L. M. (1979) Biochim. Biophys. Acta, 551, 248.

Gerristen, W. J., Van Zoelen, E. J. J., Verkleij, A. J., De Kruiff, B. and Van Deenan, L. L. M. (1979) Biochim Biophys. Acta, 551, 248.

Grandjean, J. and Laszlo, P. (1982) Biochem. Biophys. Res. Commun., 104, 1293.

Grandjean, J. and Laszlo, P. (1984) J. Am. Chem. Soc., 106, 1472.

Grell, E., Funck, Th. and Eggers, F. (1974) in Membranes: A series of advances, (ed. G. Eisenman) (New York: Marcel-Dekker).

Gupta, R. K. and Gupta, P. (1982) J. Mag. Res., 47, 344.

Hladky, S. B. (1979) Curr. Top. Membr. Transp., 12, 1.

Hober, R. (1945) Physical chemistry of cells and tissues (London: Churchill).

Hunt, G. R. A., Tipping, L. R. H. and Belmont, M. R. (1978) Biophys. Chem., 8, 341.

Hunt, G. R. A. (1980) FEBS Lett., 119, 132.

Hunt, G. R. A. and Jawaharlal, K. (1980) Biochim. Biophys. Acta, 601, 678.

Ivanov, V. T. (1975) Ann. N. Y. Acad. Sci., 264, 221.

Lau, A. L. Y. and Chan, S. I. (1975) Proc. Natl. Acad. Sci. USA, 72, 2170.

Lawaczeck, R., Blackman, D. and Kainosho, M. (1977) Biochim. Biophy. Acta, 468, 411.

Lee, Y. and Chan, S. I. (1977) Biochemistry, 16, 1303. 
O’Brien, D. F., Zumbulyadis, N., Michaels, F. M. and Ott, R. A. (1977) Proc. Natl. Acad. Sci. USA, 74, 5222. Ovchinnikov, Yu. A., Ivanov, V. T. and Shkrob, A. N. (1974) Membrane active complexones (Amsterdam: Elsevier).

Pierce, H. D., Unrau, A. M. and Oehlschlanger, A. C. (1978) Can. J. Biochem., 56, 801.

Pike, M., Simon, S., Balschi, J. A. and Springer, Jr., C. S. (1982) Proc. Natl. Acad. Sci. USA, 79, 810.

Pike, M. and Springer, Jr., C. S. (1982) J. Mag. Res., 46, 348.

Pressman, B. C. (1976) Annu. Rev. Biochem., 45, 501.

Sankaram, M. B. (1983) Structural, mechanistic and kinetic aspects of valinomycin mediated transmembrane cation transport, Ph.D. thesis, Indian Institute of Science, Bangalore.

Sankaram, M. B., Easwaran, K. R. K., Mitra, P. and Balasubramanian, D. (1984) XI International Conference on Magnetic Resonance in Biological Systems, Goa, India.

Sankaram, M. B. and Easwaran, K. R. K. (1984) in Magnetic resonance in biology and medicine, (eds G. Govil, C. L. Khetrapal, A. Saran) (Delhi: Tata-McGraw Hill).

Shastri, B. P. and Easwaran, K. R. K. (1984) Int. J. Biol. Macromol., 6, 219.

Shastri, B. P. (1985) Structural, kinetic and mechanistic aspects of carboxylic ionophore mediated transmembrane cation transport, Ph.D. thesis, Indian Institute of Science, Bangalore.

Shekar, S. C, Sankaram, M. B; and Easwaran, K. R. K. (1984) Biophys. J., (in press).

Szabo, G., Eisenman, G., Laprade, R., Ciani, S. M. and Krasne, G. (1975) Membrane; A series of advances, (ed. G. Eisenman) (New York: Marcel Dekke).

Ting, D. Z., Hagan, P. S., Chan, S. I., Doll, J. D. and Springer, Jr., C. S. (1981) Biophys. J., 34, 189.

Urry, D. W., Venkatachalam, C. M., Spilm, A., Bradley, R. J., Trapane, T. L. and Prasad, K. U. (1980) J. Membrane Biol., 55, 29.

Vishwanath, C. K. and Easwaran, K. R. K. (1981) Biochemistry, 20, 2018.

Vishwanath, C. K. and Easwaran, K. R. K. (1982) FEBS Lett., 153, 320.

Vishwanath, C. K. and Easwaran, K. R. K. (1985) J. Chem. Soc. Perkin Trans., 2, 65. 